\title{
Gamification als didaktisches Mittel in der Hochschulbildung
}

\author{
Daniel Tolks und Michael Sailer
}

\section{Zusammenfassung}

Im Rahmen des Beitrages werden die Potenziale von Gamification für den Einsatz in der Hochschulbildung verdeutlicht. Neben dem empirischen Stand der Forschung werden die Wirkungsweisen und theoretischen Modelle der Wirkung von Gamification auf Lernerfolg und Motivation skizziert. Des Weiteren werden die einzelnen Spielelemente wie Punkte, Leaderboards, Badges, Performance Graphs, Level-ups, Storytelling und Avatare und deren Wirkungsweise erläutert und auf ihre didaktische Eignung geprüft. Einige Empfehlungen für den Einsatz von Gamification in der Hochschulbildung (Designprinzipien) werden auf Basis der aktuellen empirischen Daten gegeben. Ergänzend wird die HFD Community Working Group „Netzwerk Serious Games und Gamification for Health“ vorgestellt. Der Beitrag verdeutlicht die Wirkungsweisen einzelner Spielelemente, um den Leser*innen zu ermöglichen, selbst zu prüfen, welche Spielelemente in der eigenen Lehre sinnvoll sein könnten.

\section{Tolks $(\varangle)$}

Leuphana Universität Lüneburg, Lüneburg, Deutschland

E-Mail: daniel.tolks@leuphana.de

Klinikum der Ludwig-Maximilians-Universität München, München, Deutschland

M. Sailer

Ludwig-Maximilians-Universität München, München, Deutschland

E-Mail: michael.sailer@psy.lmu.de 


\section{Schlüsselwörter}

Gamification • Hochschulbildung • Selbstbestimmungstheorie • Designprinzipien $\bullet$ Game-Design-Elemente $\bullet$ Punkte $\bullet$ Leaderboards $\bullet$

Badges • Performance Graphs • Level-up • Storytelling • Avatare

\section{Gamification}

Die Idee, die Elemente von Spielen für sinnhafte Zwecke einzusetzen, ist fast so alt wie die Menschheitsgeschichte (Tolks et al. 2018). Computerspiele sind mittlerweile stark verbreitet und werden als Kulturgut anerkannt (Deutscher Kulturrat 2018). Nach Angaben des Digitalverbands Deutschlands spielen $42 \%$ der deutschen Bevölkerung ab 14 Jahren zumindest gelegentlich, wobei das Geschlechterverhältnis beinahe ausgeglichen ist (Bitkom Research 2019). Die zu Beginn entstandenen Ressentiments und negativen Darstellungen von Computerspielen unterliegen aktuell einem Wandel. Positive Aspekte von Spielen auf Lernen sowie Verhaltens- und Einstellungsänderungen von Spielenden rücken zunehmend in den Fokus.

2006 haben Ryan und Kollegen Computerspiele auf ihre motivationalen Effekte untersucht und konnten einzelne Spielelemente und deren Wirkung auf die Motivation identifizieren (Ryan et al. 2006). Diese einzelnen GameDesign-Elemente wurden daraufhin gezielt eingesetzt, um die Motivation und die Leistung der Nutzer*innen zu steigern. Den zielgerichteten Einsatz von GameDesign-Elementen in spielfremden Kontexten bezeichnet man als Gamification (Deterding et al. 2011). Dazu gehören spielerische Elemente wie Punkte, Bestenlisten, Avatare, Level-ups, Quests (Aufgaben oder Missionen) und Badges (virtuelle Auszeichnungen). Die am häufigsten genutzten Game-Design-Elemente sind Punkte, Auszeichnungen und Bestenlisten, die in Kombination auch als PBL-Triad (Points, Badges, Leaderboards) bezeichnet werden (Bai et al. 2020).

Einige Studien stellen den Bezug zwischen verschiedenen Game-DesignElementen und der Selbstbestimmungstheorie (SDT) von Ryan und Deci her (Mekler et al. 2017; Ryan und Deci 2000; Sailer 2016). Diese definiert drei universelle psychologische Grundbedürfnisse, die das menschliche Handeln bestimmen: Kompetenzerleben, Autonomieerleben sowie das Erleben sozialer Eingebundenheit (Ryan und Deci 2000). Erste Studien im Forschungsfeld der Gamification konnten ebenfalls den Einfluss von Game-Design-Elementen auf die psychologischen Grundbedürfnisse belegen (siehe Tab. 1) (Hong und Masood 2014; Mekler et al. 2017; Sailer et al. 2017; Zainuddin et al. 2020). 
Tab. 1 Zusammenhang von psychologischen

Bedürfnissen und

Game-Design-Elementen.

(Eigene Darstellung mod. $n$.

Sailer et al. 2017; Zainuddin et al. 2020)

\begin{tabular}{l|l}
\hline Psychologisches Bedürfnis & Game-Design-Element \\
\hline Kompetenzerleben & Punkte \\
& Bestenliste \\
& Auszeichnungen (Badges) \\
& Leistungsanzeige \\
& Levelsystem \\
& Quests (Missionen, \\
& Aufgaben) \\
& Kollektionen \\
& Virtuelle Güter \\
& Freischaltung von Inhalten \\
\hline Autonomie & Profilgestaltung \\
& Avatare \\
& Freie Gestaltung der \\
& Aktivitäten \\
& Auszeichnungen (Badges) \\
& Bedeutsame Storys \\
& Quests \\
\hline Soziale Zugehörigkeit & Teams \\
& Teamevents \\
& Bestenliste \\
& Auszeichnungen (Badges) \\
& Netzwerkfunktionen \\
& (Kommentare, Likes) \\
& Epic Meaning \\
& Virtuelle Güter \\
& Freischaltung von Inhalten \\
\hline &
\end{tabular}

Studien und Forschungsarbeiten konnten einige der positiven Effekte von Gamification bestätigen. So kann Gamification die Motivation stärken (Sailer et al. 2017), das User-Engagement und auch soziale Interaktionen erhöhen (Hamari und Koivisto 2013) sowie den positiven Wettbewerb fördern (Kapp 2012). Gamification ist leicht zugänglich (King et al. 2013; Lister et al. 2014), lässt sich in den Alltag integrieren (Deterding 2015; Munson et al. 2015) und ist den meisten Nutzer*innen bereits durch das Medium Spiel vertraut (Tolks et al. 2018). Im Vergleich zur Entwicklung von digitalen Serious Games und Lernspielen ist der Einsatz von Gamification weitaus günstiger (Munson et al. 2015) und niedrigschwelliger. Der Einsatz von Gamification setzt allerdings einige Aspekte voraus, die beachtet werden müssen.

Die Bedeutung von Gamification in der Wissenschaft spiegelt sich auch in der wachsenden Zahl veröffentlichter Arbeiten wider (Bai et al. 2020; Koivisto und 
Tab. 2 Liste von Gamification-Plug-ins in Moodle. (Eigene Darstellung der Inhalte von Singh [2017/2019], (LMS Pulse)

\begin{tabular}{l|l}
\hline Name & Inhalt \\
\hline Level-up & $\begin{array}{l}\text { Die Nutzer*innen können, basierend auf ihren Aktionen, } \\
\text { Erfahrungspunkte innerhalb des Kurses sammeln. Die aktuellen } \\
\text { Punkte werden in einem Block angezeigt und die Lernenden } \\
\text { steigen ins nächste Level auf }\end{array}$ \\
\hline Game & $\begin{array}{l}\text { Mit dem Game-Plug-in können interaktive Spiele mithilfe von } \\
\text { Fragen, Glossaren und Quiz erstellt werden. Es enthält acht Spiele: } \\
\text { Henker, Kreuzworträtsel, Kryptex, Millionär, Sudoku, Schlangen } \\
\text { und Leitern, das versteckte Bild sowie das Buch mit Fragen }\end{array}$ \\
\hline Quizventure & $\begin{array}{l}\text { Die Fragen aus dem Kurs erscheinen in Form eines Schiffes und } \\
\text { die Nutzer*innen müssen auf die richtigen Antworten schießen }\end{array}$ \\
\hline Stash & $\begin{array}{l}\text { Die Lehrenden können Inhalte in Form eines ,Schatzes“ in der } \\
\text { Kursumgebung verstecken und von den Nutzer*innen freischalten } \\
\text { lassen }\end{array}$ \\
\hline Badge & $\begin{array}{l}\text { Badges können anhand einer Vielzahl ausgewählter Kriterien } \\
\text { vergeben und im Profil der teilnehmenden Person angezeigt } \\
\text { werden. Die Erstellung lässt viel Freiraum für Kreativität }\end{array}$ \\
\hline Mootrain/Mootivated & $\begin{array}{l}\text { Die Nutzer*innen werden mit virtuellen Münzen für das Lernen in } \\
\text { Moodle belohnt. Die Lernenden können diese Münzen verwenden, } \\
\text { um den Avatar mit verschiedenen Gegenständen zu modifizieren }\end{array}$ \\
\hline
\end{tabular}

Hamari 2014; Ryan et al. 2006; Sailer und Homner 2020; Zainuddin et al. 2020). Insbesondere im Gesundheitsbereich gibt es zahlreiche Studien, die die Effekte des Einsatzes von Gamification auf Motivation, Leistung, Engagement und den Gesundheits- sowie Wellbeingstatus nachweisen konnten (Johnson et al. 2016; Schmidt-Kraepelin et al. 2018; Tolks et al. 2020).

Einem Großteil der Studien fehlt derzeit der Bezug zu theoretischen Grundlagen, wie beispielsweise Lerntheorien, außerdem sind die unterschiedlichen spielerischen Ansätze nicht präzise differenziert (Bai et al. 2020; Baranowski 2018; Breuer und Tolks 2018). Das Anwendungs- und Forschungsfeld ist sehr divers und interprofessionell aufgestellt, was zu einer Fragmentierung der Wissensbasis geführt hat (Schmidt-Kraepelin et al. 2018). 


\section{Game-Design-Elemente}

Im Folgenden werden die gängigsten Game-Design-Elemente und deren Wirkung auf Motivation und Leistung beschrieben.

\section{Punkte}

Bei einem Punktesystem werden die Aktivitäten der Nutzer*innen mit Punkten belohnt. Punkte ermöglichen ein direktes und kontinuierliches Feedback und dienen als Belohnungssystem (Sailer et al. 2014). Durch die kontinuierliche Sammlung von Punkten können die Nutzer*innen und die Lehrenden eine Rückmeldung über den Fortschritt erhalten und beispielsweise den Lernprozess besser abbilden (Werbach und Hunter 2012).

\section{Bestenlisten}

In einer Bestenliste werden die Nutzer*innen in einer Liste, abhängig von den erhaltenen Punkten oder Badges, in direkten Vergleich zu den anderen Teilnehmer*innen gesetzt. Durch den Einsatz einer Bestenliste werden die Leistungen transparent (Costa et al. 2013).

\section{Badges}

Bei Badges handelt es sich um virtuelle Auszeichnungen, ähnlich dem Ordenoder Medaillensystem im Sport oder Militär. Diese Auszeichnungen symbolisieren eine erbrachte Leistung (Zicherman und Cunningham 2011). Sie können als Symbol für Status oder der Zugehörigkeit zu einer Bestengruppe wahrgenommen werden (Antin und Churchill 2011). Badges können als erreichbares Ziel und Steuerungselement (Zicherman und Cunningham 2011) dienen und so das Verhalten beeinflussen (Wang und Sun 2011).

\section{Progress Bars}

Fortschrittsanzeigen liefern Informationen über die Leistung der Nutzer*innen im Vergleich zu ihrer vorherigen Leistung (Sailer et al. 2014). Der Progress Bar bildet dabei nur die individuelle Leistung der Nutzer*innen ab. Durch die grafische Darstellung der Leistung kann die teilnehmende Person jederzeit ihren Fortschritt einsehen und zudem sehen, wie viele Aktivitäten benötigt werden, um die nächste Stufe zu erreichen, wenn ein Levelsystem hinterlegt ist (Sailer et al. 2014).

\section{Avatare}

Bei Avataren handelt es sich um virtuelle Stellvertreter der Nutzer*innen. Avatare können dabei jede beliebige Form annehmen. Es können vorgefertigte Charaktere 
sein oder virtuell erstellte Abbilder der Nutzer*innen (Kapp 2012). Avatare dienen als Unterscheidungsmerkmal und können einen Rollenwechsel ermöglichen (Werbach und Hunter 2012). Durch die Nutzung spezieller Avatare kann sich die Person einer Community zugehörig fühlen (Annetta 2010).

\section{Level-up}

Bei einem Level-up steigen die Spieler*innen auf eine nächsthöhere Stufe. Ein Stufenaufstieg ist in Computerspielen meistens mit einer Verbesserung der Attribute und einem Zugewinn an neuen Fähig- und Fertigkeiten verbunden. Diese Levelaufstiege können auch im Bereich Gamification eingesetzt werden, um Nutzer*innen zu motivieren, sich länger mit Inhalten zu beschäftigen. Dabei ist zu beachten, dass auch in Lernumgebungen der Levelaufstieg einen wahrnehmbaren Nutzen haben sollte (Bai et al. 2020).

\section{Storytelling}

Erzählstrukturen und Hintergrundgeschichten sind Kernelemente von Computerspielen, die dazu führen können, sich länger mit dem Spiel zu beschäftigen und mit dem Spielerlebnis Bedeutsamkeit zu verbinden. Gute Hintergrundgeschichten können auch bei der Anwendung von Gamification eingesetzt werden. Die narrativen Elemente sind unabhängig von der Performanz der Nutzer*innen. Sie können eine veränderte Bedeutung evozieren, dabei aber Analogien zur „echten Welt“ herstellen. Storytelling ist vor allem dann geeignet, wenn die Realität als langweilig wahrgenommen wird und/oder die narrativen Komponenten einen Bezug zu persönlichen Interessen aufweisen (Nicholson 2015). Im Bereich Gamification stellt die Verknüpfung einer bedeutsamen Geschichte mit einer gamifizierten Lernumgebung eine große Herausforderung dar, da die Geschichte über eine bereits bestehende Struktur ,übergestülpt“ werden muss (Narrative Overlay) (Sailer et al. 2017).

\section{Quests}

Bei Quests (Missionen, Aufgaben) handelt es sich um Aufgaben, die vom Spiel gestellt werden und die die Nutzer*innen erfüllen können oder müssen. Diese Aufgaben können als Steuerungselement oder als Einführung in eine Thematik genutzt werden. In Lernumgebungen lassen sich Quests dafür einsetzen, eng umfasste und vorgeschriebene Lernpfade im Sinne der „Scaffolded Instruction“ (Hogan und Pressley 1997) vorzugeben. In Computerspielen müssen die Spieler*innen das bereits erlernte Wissen sowie die Fertig- und Fähigkeiten gegen herausfordernde Gegner*innen nutzen. Es handelt sich um eine Prüfung des 
bislang erworbenen Wissens bzw. der erworbenen Fertig- und Fähigkeiten. Dieses Prinzip kann auch auf Lernumgebungen übertragen werden. Quests werden insbesondere dann herausfordernd, wenn ein erfolgreicher Abschluss nur durch den gemeinsamen Einsatz als Team erreicht werden kann. Der Aufgabe wird dadurch eine höhere Bedeutsamkeit zugeschrieben, was auch als „Epic Meaning“ bezeichnet wird (Koch und Ott 2012).

\section{Gamification im Lernkontext}

Im Rahmen von Gamification im Bildungskontext werden Lernprozesse mit Game Design-Elementen angereichert, um eine veränderte Version dieses Lernprozesses zu erhalten, den die Lernenden als spielerisch wahrnehmen (Landers et al. 2018). Der Einsatz von Gamification in Bildungskontexten gehört zu den am stärksten repräsentierten Forschungsbereichen innerhalb der Literatur zu Gamification. Häufige Ziele von Gamification im Bildungskontext sind die Förderung von unterschiedlichen Wissensarten, die Steigerung von (Lern-)Motivation und der Erwerb von Fähigkeiten und Kompetenzen (Sailer und Homner 2020).

Trotz der oftmals fehlenden theoretischen Fundierung empirischer Studien zum Thema Gamification und Lernerfolg, gibt es theoretische Ansätze, die sich den Effekten von Gamification auf den Lernerfolg widmen, wie zum Beispiel die „Theory of Gamified Learning“ (Landers und Landers 2014). Teile dieser Theorie sagen aus, dass Game-Design-Elemente lernrelevante Aktivitäten fördern können, die wiederum in positivem Zusammenhang mit dem Lernerfolg stehen. Lernrelevante Aktivitäten beispielsweise stellen eine tiefergehende kognitive Verarbeitung dar (Johnson et al. 2016; Turan et al. 2016; Sailer und Homner 2020; Zainuddin et al. 2020). Das ICAP-Modell (Chi und Wylie 2014) postuliert eine Taxonomie von Lernaktivitäten und besagt, dass Lernen umso effektiver und nachhaltiger ist, je tiefer die Lernenden kognitiv involviert sind und je aktiver sie sich engagieren. Vor allem die interaktiven und konstruktiven Lernaktivitäten sind für den Wissenserwerb von Lernenden entscheidend, da diese mit höheren kognitiven Investitionen in das Lernen verbunden sind.

Laut einer Metaanalyse von Sailer und Homner hat Gamification positive Effekte auf kognitive, motivationale und verhaltensbezogene Lernergebnisse (Sailer und Homner 2020). Bai et al. konnten in einer Metaanalyse ebenfalls nachweisen, dass der Einsatz von Gamification zu besseren Lernergebnissen im Vergleich zu Lernangeboten ohne Gamification führt (Bai et al. 2020). 
Der Einsatz von Gamification in speziellen Settings wie zum Beispiel im Schulunterricht, in der Ausbildung von Gesundheitsberufen und in der medizinischen Ausbildung zeigt ebenfalls positive Ergebnisse (Nevin et al. 2014; Sailer et al. 2019; Tolks et al. 2020).

\section{$4 \quad$ Gamification in der Hochschulbildung}

Im Zuge der Digitalisierung und des digitalen Wandels wurde eine Vielzahl an Möglichkeiten geschaffen, online zu lernen, sei es im Rahmen der Hochschulbildung, in der Schule, in Unternehmen, in der Fort- und Weiterbildung sowie im privaten Bereich (Kuhn et al. 2017). Oftmals scheitert der Lernerfolg der Projekte an mangelnder Motivation der Teilnehmer*innen, insbesondere wenn es sich um reine Onlinelernumgebungen handelt und sich die Nutzer*innen die Inhalte alleine und ohne die Unterstützung von Peers aneignen müssen. Diese Situation herrscht besonders in der Hochschulbildung vor. Zusätzlich ist es vor allem in der Hochschulbildung relevant, Lernumgebungen für sehr große Gruppen von Studierenden zu entwickeln (Sailer und Sailer 2020).

Studien zeigen, dass Gamification den Lernprozess sehr unterstützen und zu einer Motivations- und Leistungssteigerung der Studierenden führen kann. Der Einsatz von Gamification hat zudem das Potenzial, das Engagement der Studierenden zu erhöhen, indem Terminologie und Konzepte verwendet werden, mit denen sie positive Assoziationen verbinden. Die Lernenden erhalten schnelles Feedback zu ihren Fortschritten sowie sofortige positive Verstärkung, was sie bereits aus Videospielen und Apps kennen, die Teil ihrer Lebenswelt geworden sind. Motivation und Engagement können durch spielerische Anreize gesteigert werden, sodass die Studierenden die positiven Auswirkungen auf ihren Lernerfolg schneller erkennen und Vertrauen aufbauen. Ebenso kann so ein konstruktiver Wettbewerb zwischen den Nutzer*innen gefördert werden.

Bai et al. (2020) konnten vier Aspekte identifizieren, die Studierende positiv mit Gamification verbinden: 1) Gamification kann den Enthusiasmus fördern, 2) Gamification ermöglicht Feedback auf die eigene Leistung, 3) Gamification kann das Bedürfnis der Lernenden nach Anerkennung erfüllen und 4) Gamification ist förderlich für die Zielerreichung. Zwei negative Aspekte im Zusammenhang mit Gamification konnten ebenfalls identifiziert werden: 1) Gamification hat keinen konkreten Nutzen für die Nutzer*innen und 2) der Einsatz von Gamification kann Unsicherheit und Neid generieren (Bai et al. 2020). 
Gamification kann mittlerweile mit überschaubarem Aufwand in bestehende Lern-Management-Systeme (LMS) integriert werden. Eine Auswahl an MoodlePlug-ins mit dazugehörigen offiziellen Beschreibungen werden im Folgenden kurz beschrieben.

\section{Designprinzipien für den Einsatz von Gamification}

Basierend auf den aktuellsten empirischen Befunden sollen im Folgenden Designprinzipien für den Einsatz von Gamification in der Hochschulbildung aufgeführt werden. Diese sollen dazu dienen, den Leser*innen eine Anleitung für den Einsatz von Gamification in den eigenen Projekten zu geben.

\section{Kombination von mehreren Game-Design-Elementen}

Um möglichst alle psychologischen Grundbedürfnisse der Nutzer*innen zu avisieren, kann es sinnvoll sein, mehrere Game-Design-Elemente zu nutzen (Ryan et al. 2006; Ryan und Deci 2000; Sailer et al. 2017). Tab. 1 zeigt dabei, welche GameDesign-Elemente verwendet werden sollten. Zudem existieren einige häufig angewandte Kombinationen. In einer Studie konnte allerdings auch nachgewiesen werden, dass der Einsatz von Badges Einfluss auf alle drei psychologischen Grundbedürfnisse haben kann (Xi und Hamari 2019). So ist es bei Umsetzungen bestimmter Game-Design-Elemente (zum Beispiel Badges) prinzipiell möglich, alle drei psychologischen Grundbedürfnisse anzusprechen.

\section{Aktivierendes Lernen ermöglichen}

Laut Snyder, Chi und Wylie stellen aktivierende Lehr-Lern-Methoden die wichtigsten Faktoren für einen sinnhaften Lernprozess dar (Chi und Wylie 2014; Snyder 2003). Statt Passivität erfordert Active Learning die aktive Einbeziehung der Studierenden in den Lernprozess, was eine intensivere Lernerfahrung zur Folge haben soll, die über bloße Reproduktion hinausgeht (Struyven et al. 2006). Active Learning führt $\mathrm{zu}$ einem nachhaltigeren Wissenserwerb, fördert die Fähigkeit, Probleme zu lösen, und wirkt sich positiv auf die Lernmotivation aus (Snyder 2003). Diese Aktivierung kann in der Hochschulbildung durch den Einsatz von gamifizierten Tools wie Quizprogrammen (zum Beispiel Kahoot!) oder Audience-Response-Systemen unterstützt werden. Durch den Wettbewerb mit Punkten und Leaderboards kann die Aktivierung der Studierenden über die komplette Lerneinheit hinweg aufrechterhalten werden (Fuster-Guilló et al. 2019). 


\section{Theoretische Fundierung}

Der Einsatz von Gamification sollte auf einer theoretischen Grundlage basieren. Landers et al. (2018) haben die mangelnde theoretische Fundierung von Studien im Bereich Gamification beschrieben (Landers et al. 2018). Die am häufigsten verwendete Theorie in der Hochschulbildung stellt die Selbstbestimmungstheorie dar (Zainuddin et al. 2020). In der Hochschulbildung ist insbesondere darauf zu achten, dass die gamifizierten Lernkonzepte auf den Lerntheorien wie der „Theory of Gamified Learning“ (Landers und Landers 2014) oder dem ICAP Modell basieren (Chi und Wylie 2014).

\section{Abwägung beim Einsatz von kompetitiven Game-Design-Elementen}

Die kompetitiven Bestandteile einiger Game-Design-Elemente, insbesondere von Leaderboards, sollten immer mit Bedacht eingesetzt werden. Einerseits kann sozialer Druck zwar konstruktiv wirken und das Engagement erhöhen (Burguillo 2010), andererseits können verschiedene Motivationsmuster bei unterschiedlichen Lerner*innen entstehen (Sailer 2016). In der Metaanalyse von Sailer und Homner ließen sich keine eindeutigen positiven Effekte beim Einsatz von Wettbewerben nachweisen (Sailer und Homner 2020). Zudem können Wettkämpfe zu einem hohen Stresslevel führen, welches wiederum einen negativen Effekt auf den Lernerfolg haben kann (Friedlander et al. 2011). Wettkampf hat vor allem dann Potenzial, zu höherem Engagement und zu höherer Motivation zu führen, wenn sich alle Lernende auf dem gleichen Leistungsniveau befinden (Malone und Lepper 1987). Damit die kompetitiven Prozesse besser funktionieren und Chancengleichheit gewahrt werden kann, sollten sich daher alle Teilnehmenden auf demselben Level (Vorwissen, Ausbildungsstand) befinden und möglichst zeitgleich beginnen (Landers und Landers 2014). Durch kooperative Aufgaben und die Bildung von Teams kann der Leistungsdruck für das Individuum reduziert werden (Sailer und Homner 2020).

\section{Erstellung von Teams}

Einerseits kann die gemeinsame Bearbeitung von Zielen zu gegenseitiger Unterstützung, Leistungssteigerung oder Kooperation und Motivation beitragen, andererseits können einzelne Teammitglieder aber auch in Konflikte und Wettkampf eintreten (Burguillo 2010; Gustafsson et al. 2009; Kapp 2012). Gamification kann als Katalysator für kollaboratives Lernen fungieren und die gemeinsame Bearbeitung von Zielen fördern (Singhal et al. 2019). Sailer und Homner konnten vielversprechende Effekte nachweisen, wenn Wettbewerb mit Kooperation gekoppelt wird (Sailer und Homner 2020). 
Durch den Einsatz von Teams kann ein Scheitern nicht als persönliches, sondern als gemeinsames Scheitern wahrgenommen werden. Zudem kann dieses Scheitern $\mathrm{zu}$ einem neuen Versuch motivieren. Ein weiterer positiver Aspekt ist das Konzept des „Epic Meanings“ (Koch und Ott 2012). Es beschreibt den Glauben an ein höheres Ziel, das alleine nicht zu bewältigen scheint und nur gemeinsam mit weiteren Mitstreiter*innen geschafft werden kann. Ein Beispiel wäre die Überwindung einer großen Laufstrecke als Firmenevent, wobei die Mitarbeiter*innen gemeinsam die Gesamtstrecke erlaufen müssen.

\section{Einsatz von Storytelling}

Der Einsatz von Storytelling und narrativen Elementen kann die Anwendung des erworbenen Wissens fördern und situiertes Lernen ermöglichen (Petko 2008). Storytelling-Elemente werden als relevanter empfunden, wenn der Lernkontext als weniger interessant wahrgenommen wird oder ein Bezug zu den persönlichen Interessen hergestellt wird (Nicholson 2015). Die Einflussnahme auf und die Interaktion mit der Storyline ist eines der bedeutsamsten Elemente von Computerspielen. Die Einflussnahme auf die Geschichte kann die Handlungen der Nutzer*innen mit Bedeutsamkeit versehen (Mohan et al. 2007; Sailer und Homner 2020). Die Nutzung von narrativen Elementen ist ein vielversprechender Gamification-Ansatz, der zu positiven Effekten auf den Lernerfolg führen kann (Sailer und Homner 2020).

\section{Vernetzen von Gamification und Inverted Classroom}

Der Einsatz von Gamification zur Unterstützung der Inverted-Classroom-Methode scheint gut geeignet zu sein. Zum einen kann die Onlineselbstlernphase durch die Anreicherung von Game-Design-Elementen unterstützt werden (Huang und Hew 2018; Sanchez et al. 2020). Zum anderen kann auch die Präsenzphase durch den Einsatz von gamifizierten Quizformaten und Audience-Response-Systemen angereichert werden (Sailer und Sailer 2020; Tolks et al. 2020; Zainuddin et al. 2020). In einer Studie gaben die Studierenden an, dass der Einsatz von Leaderboards in Kombination mit der Inverted Classroom Model als am motivierendsten wahrgenommen wurde (Aldemir et al. 2018).

\section{Nachhaltigkeit}

Bei der Entwicklung von gamifizierten Lernumgebungen sollte auf Folgendes geachtet werden: Zum einen sollten die evozierten Effekte von Gamification möglichst lange aufrechterhalten werden und zum anderen sollte darauf geachtet werden, dass diese neu entwickelten Lernumgebungen mehrmals genutzt werden können. Der initiale Mehraufwand zur Entwicklung von innovativen, interaktiven 
und gamifizierten Lernumgebungen stellt eine der größten Barrieren für die Lehrenden dar (Hammer und Lee 2011). In der Hochschulbildung bieten sich daher Lehrveranstaltungen an, die fortlaufend durchgeführt werden, wie beispielsweise Pflichtveranstaltungen.

\section{Differenzierung von Gamification}

Wie in einigen Studien angemerkt, werden oftmals mehrere spielbasierte Ansätze nicht trennscharf differenziert. Konzepte wie Game-based Learning, Serious Games, Lern- und Planspiele werden selbst in Übersichtsstudien nicht klar getrennt. Die Folge ist eine große Heterogenität der Studienqualität, deren Ergebnisse oftmals nicht aussagekräftig sind (Bai et al. 2020; Baranowski 2018; Tolks und Lampert 2016). Wenn der Einsatz von Gamification geplant wird, muss im Vorfeld geprüft werden, welche spielbasierten Methoden für welche Zwecke eingesetzt werden sollen.

\section{Entwicklung von neuen Game-Design-Elementen}

Aktuell werden immer wieder lediglich die Elemente der PBL-Triade genutzt. Zainuddin et al. (2020) gaben in ihrer Studie zu bedenken, dass zwar Leaderboards von den meisten Studierenden als sehr motivierend wahrgenommen wurden, aber der Einsatz von neuen Game-Design-Elementen, wie zum Beispiel die Möglichkeit, andere Nutzer*innen zu beschenken, eine hohe soziale Verbundenheit zur Folge hatte. Sie rufen daher dazu auf, nicht nur die PBL-Triad zu nutzen, sondern auch neue Game-Design-Elemente zu entwickeln (Zainuddin et al. 2020).

\section{Anpassung an die jeweilige Zielgruppe}

Bei der Entwicklung von gamifizierten Lernumgebungen muss die Zielgruppe berücksichtigt werden. So existieren unterschiedliche Spieler*innentypen (Koivisto und Hamari 2014). Es ist wichtig, die Bedürfnisse der Zielgruppe frühzeitig zu erfassen und gezielt Game-Design-Elemente einzusetzen, um die vorliegenden Bedürfnisse und Probleme zu antizipieren (Hallifax et al. 2019). Besteht beispielsweise eine geringe Motivation bei den Studierenden, sollten gezielt Game-Design-Elemente eingesetzt werden, die sich positiv auf die Motivation auswirken. Besteht die Problematik, dass die Studierenden sich nicht ausreichend lange mit Inhalten beschäftigen, sollten Game-Design-Elemente eingesetzt werden, die das zeitliche Engagement erhöhen. Beispielsweise könnten Badges vergeben werden, wenn alle Lernvideos angeschaut wurden. 
Game-Design-Elemente wirken nicht immer gleich

Als letzter Punkt soll hier dargelegt werden, dass pauschale Wirksamkeitserwartungen beim Einsatz von Gamification nicht immer erfüllt werden. Nicht jedes Game-Design-Element entfaltet die gleiche Wirkung, sondern ist abhängig von Design und Umsetzung. So ist die Wirkung von Badges davon abhängig, wie oft sie vorkommen und wie schwer sie zu erreichen sind (Groening und Binnewies 2019). Schwere Quests oder Quizfragen können dazu führen, dass das Kompetenzerleben des Individuums oder des Teams abnimmt (Zainuddin 2018; Sailer und Sailer 2020). Die auch im Gamebereich bekannte Problematik des passgenauen Zusammenspiels von Herausforderung und Bewältigung betrifft auch den Einsatz von Gamification. Die Möglichkeit des Flow-Erlebens in Computerspielen (Admiraal et al. 2011) ist eine der Besonderheiten von Computerspielen und gleichzeitig auch eine der größten Herausforderungen des Gamedesigns.

\section{Ausblick}

Um die Aktivitäten im Bereich Gamification und Serious Games im deutschsprachigen Raum zu intensivieren, wurde 2019 eine Community Working Group gegründet, die im Folgenden kurz vorgestellt werden soll.

Im Rahmen der Community Working Group des Hochschulforums Digitalisierung wird aktuell die Gründung des Netzwerks „Serious Games und Gamification for Health" in Deutschland geplant. ${ }^{1}$ Die grundlegende Zielsetzung des Netzwerks basiert auf zwei Ideen: Zum einen sollen möglichst alle Akteur*innen im Bereich Serious Games und Gamification for Health in Deutschland, Schweiz und Österreich zusammengebracht werden, um Erfahrungen und Expert*innenwissen auszutauschen und Aktivitäten zu bündeln und im Idealfall gemeinsame Anträge zu erstellen. Zum anderen will das Netzwerk wissenschaftlich fundierte Informationen zum oben genannten Themenkomplex bereitstellen. Aktuell wird eine Website aufgebaut, auf der sich interessierte Personen Informationen über die aktuelle Studienlage, neueste Entwicklungen und Veranstaltungen informieren können. In einer weiteren Phase soll geprüft werden, inwieweit die Ausweitung des Netzwerkes auf andere Themen- und Handlungsfelder außerhalb des Gesundheitsbereichs (wie zum Beispiel Hochschule, Schule) möglich ist.

Betrachtet man die derzeitigen Entwicklungen, besonders im Bereich Gamification, entsteht schnell der Eindruck eines Hypes. Daher stellt sich die Frage,

\footnotetext{
${ }^{1}$ Siehe: https://hochschulforumdigitalisierung.de/de/cwg-seriousgameshealth. Zugegriffen: 04.11.2020.
} 
inwieweit es Gamification auch zukünftig noch geben wird. Laut dem Gartner Hype Cycle for Education hat Gamification bereits die ersten drei Stufen des Hypeprozesses durchlaufen und befindet sich in der Konsolidierungs- und Implementierungsphase (Gartner Research 2016). Durch die zunehmend reflektierte und differenzierte Diskussion über den Einsatz von spielerischen Elementen bleibt zu hoffen, dass in Zukunft neue Studien und Projekte durchgeführt werden und die empirische Datenlage weiter präzisiert wird. Wenn sich diese positiven Entwicklungen auch in Zukunft weiterführen lassen, wird der Einsatz von Gamification in der Hochschulbildung einen wichtigen Anteil bei der strukturierten und effizienten Entwicklung von interaktiven Lernprozessen und Lernumgebungen haben.

\section{Literatur}

Admiraal, W., Huizenga, J., Akkerman, S., \& Dam, G. ten. (2011). The concept of flow in collaborative game-based learning. Computers in Human Behavior, 27(3), 1185-1194 https://doi.org/10.1016/j.chb.2010.12.013.

Aldemir, T., Celik, B., \& Kaplan, G. (2018). A qualitative investigation of student perceptions of game elements in a gamified course. Computers in Human Behavior, 78, 235-254 https://doi.org/10.1016/j.chb.2017.10.001.

Annetta, L. A. (2010). The "I's" Have it: A framework for serious educational game design. Review of General Psychology, 14(2), 105-112. https://doi.org/10.1037/a0018985.

Antin, J., \& Churchill, E. F. (2011). Badges in social media: A social psychological perspective. [Vortrag] In Conference on Human Factors in Computing Systems (CHI) 2011 Gamification Workshop Proceedings. New York: ACM.

Bai, S., Hew, K. F., \& Huang, B. (2020). Does gamification improve student learning outcome? Evidence from a meta-analysis and synthesis of qualitative data in educational contexts. Educational Research Review, 30, (100322). https://doi.org/10.1016/j.edurev. 2020.100322.

Baranowski, T. (2018). Games for health research - Past, present, and future. Prävention und Gesundheitsförderung, 13(4), 333-336 https://doi.org/10.1007/s11553-018-0657-y.

Bitkom Research. (2019). Die gaming-trends 2019. Berlin: Bitkom.

Breuer, J., \& Tolks, D. (2018). Grenzen von „Serious Games for Health“. Prävention und Gesundheitsförderung, 13(4), 327-332. https://doi.org/10.1007/s11553-018-0654-1.

Burguillo, J. C. (2010). Using game theory and competition-based learning to stimulate student motivation and performance. Computers \& Education, 55(2), 566-575.

Chi, M. T. H., \& Wylie, R. (2014). The ICAP framework: Linking cognitive engagement to active learning outcomes. Educational Psychologist, 49(4), 219-243. https://doi.org/10. 1080/00461520.2014.965823.

Costa, J. P., Wehbe, R. R., Robb, J., \& Nacke, L. E. (2013). Time's up: Studying leaderboards for engaging punctual behaviour. In Proceedings of the First International Conference on Gameful Design, Research, and Applications (S. 26-33). Toronto, Ontario: Association for Computing Machinery. https://doi.org/10.1145/2583008.2583012. 
Deterding, S. (2015). The lens of intrinsic skill atoms: A method for gameful design. Human-Computer Interaction, 30(3-4), 294-335. https://doi.org/10.1080/07370024. 2014.993471.

Deterding, S., Dixon, D., Khaled, R., \& Nacke, L. (2011). From game design elements to gamefulness: Defining gamification. In Proceedings of the 15th international academic MindTrek conference: Envisioning future media environments (S. 9-15). Tampere: ACM.

Deutscher Kulturrat. (2018). Kulturgut Computerspiele. https://www.kulturrat.de/thema/kul turgut-computerspiele/. Zugegriffen: 11. Juli 2018.

Friedlander, M. L., Escudero, V., Heatherington, L., \& Diamond, G. M. (2011). Alliance in couple and family therapy. Psychotherapy, 48(1), 25.

Fuster-Guilló, A., Pertegal-Felices, M. L., Jimeno-Morenilla, A., Azorín-López, J., RicoSoliveres, M. L., \& Restrepo-Calle, F. (2019). Evaluating impact on motivation and academic performance of a game-based learning experience using kahoot. Frontiers in Psychology, 10, 2843. https://doi.org/10.3389/fpsyg.2019.02843.

Gartner Research. (2016, Juli). Hype cycle for education, 2016. Gartner. https://www.gartner. com/en/documents/3364119/hype-cycle-for-education-2016. Zugegriffen: 16. Nov. 2020.

Groening, C., \& Binnewies, C. (2019). "Achievement unlocked!" - The impact of digital achievements as a gamification element on motivation and performance. Computers in Human Behavior, 97, 151-166 https://doi.org/10.1016/j.chb.2019.02.026.

Gustafsson, A., Katzeff, C., \& Bang, M. (2009). Evaluation of a pervasive game for domestic energy engagement among teenagers. Computers in Entertainment, 7(4), 54.

Hallifax, S., Serna, A., Marty, J.-C., Lavoué, G., \& Lavoué, E. (2019). Factors to consider for tailored gamification. In Proceedings of the Annual Symposium on Computer-Human Interaction in Play. (S. 559-572) New York: Association for Computing Machinery. https://doi.org/10.1145/3311350.3347167.

Hamari, J., \& Koivisto, J. (2013). Social motivations to use gamification: An empirical study of gamifying exercise. In Proceedings of European Conference on Information Systems. (S. 105). New York: Elsevier.

Hammer, J., \& Lee, J. (2011). Gamification in education: What, how, why bother. Academic Exchange Quarterly, 15(2), 146.

Hogan, K. E., \& Pressley, M. E. (1997). Scaffolding student learning: Instructional approaches and issues. Cambridge: Brookline Books.

Hong, G. Y., \& Masood, M. (2014). Effects of gamification on lower secondary school students' motivation and engagement. International Journal of Social, Education, Economics and Management Engineering, 8(12), 3483-3490.

Huang, B., \& Hew, K. F. (2018). Implementing a theory-driven gamification model in higher education flipped courses: Effects on out-of-class activity completion and quality of artifacts. Computers \& Education, 125, 254-272.

Johnson, D., Deterding, S., Kuhn, K.-A., Staneva, A., Stoyanov, S., \& Hides, L. (2016). Gamification for health and wellbeing: A systematic review of the literature. Internet Interventions, 6, 89-106.

Kapp, K. M. (2012). The gamification of learning and instruction: Game-based methods and strategies for training and education. San Francisco: John Wiley \& Sons.

King, D., Greaves, F., Exeter, C., \& Darzi, A. (2013). „Gamification“: Influencing health behaviours with games. Journal of the Royal Society of Medicine, 106(3), 76-78 https:// doi.org/10.1177/0141076813480996. 
Koch, M., \& Ott, F. (2012). Gamification - Steigerung der Nutzungsmotivation durch Spielkonzepte. Soziotechnische Integration.

Koivisto, J., \& Hamari, J. (2014). Demographic differences in perceived benefits from gamification. Computers in Human Behavior, 35, 179-188. https://doi.org/10.1016/j.chb.2014. 03.007.

Kuhn, S., Frankenhauser, S., \& Tolks, D. (2017). Digitale Lehr- und Lernangebote in der medizinischen Ausbildung. Bundesgesundheitsblatt - Gesundheitsforschung - Gesundheitsschutz, (S. 1-9). https://doi.org/10.1007/s00103-017-2673-z.

Landers, R. N., Auer, E. M., Collmus, A. B., \& Armstrong, M. B. (2018). Gamification science, its history and future: Definitions and a research agenda. Simulation \& Gaming, 49(3), 315-337 https://doi.org/10.1177/1046878118774385.

Landers, R. N., \& Landers, A. K. (2014). An empirical test of the theory of gamified learning: The effect of leaderboards on time-on-task and academic performance. Simulation \& Gaming, 45(6), 769-785. https://doi.org/10.1177/1046878114563662.

Lister, C., West, J. H., Cannon, B., Sax, T., \& Brodegard, D. (2014). Just a fad? Gamification in health and fitness apps. JMIR Serious Games, 2(2), e9. https://doi.org/10.2196/games. 3413.

Mekler, E. D., Brühlmann, F., Tuch, A. N., \& Opwis, K. (2017). Towards understanding the effects of individual gamification elements on intrinsic motivation and performance. Computers in Human Behavior, 71, 525-534. https://doi.org/10.1016/j.chb.2015.08.048.

Mohan, V., Sandeep, S., Deepa, M., Gokulakrishnan, K., Datta, M., \& Deepa, R. (2007). A diabetes risk score helps identify metabolic syndrome and cardiovascular risk in indians - The Chennai Urban Rural Epidemiology Study (CURES-38). Diabetes, Obesity \& Metabolism, 9(3), 337-343. https://doi.org/10.1111/j.1463-1326.2006.00612.x.

Munson, S., Poole, E., Perry, D., \& Peyton, T. (2015). Gamification and Health. In S. Walz (Hrsg.), The gameful world: Approaches, issues, applications (S. 597-623). Cambridge: MIT Press.

Nevin, C. R., Westfall, A. O., Rodriguez, J. M., Dempsey, D. M., Cherrington, A., Roy, B., Patel, M., \& Willig, J. H. (2014). Gamification as a tool for enhancing graduate medical education. Postgraduate Medical Journal, 90(1070), 685-693.

Nicholson, S. (2015). A RECIPE for meaningful gamification. In T. Reiners \& L. C. Wood (Hrsg.), Gamification in education and business (S. 1-20). Cham: Springer International Publishing. https://doi.org/10.1007/978-3-319-10208-5_1.

Petko, D. (2008). Unterrichten mit Computerspielen Didaktische Potenziale und Ansätze für den gezielten Einsatz in Schule und Ausbildung. MedienPädagogik: Zeitschrift für Theorie und Praxis der Medienbildung, 15, 1-15.

Ryan, R. M., \& Deci, E. L. (2000). Self-determination theory and the facilitation of intrinsic motivation, social development, and well-being. American Psychologist, 55(1), 68.

Ryan, R. M., Rigby, C. S., \& Przybylski, A. (2006). The motivational pull of video games: A self-determination theory approach. Motivation and Emotion, 30(4), 344-360.

Sailer, M. (2016). Die Wirkung von Gamification auf Motivation und Leistung: Empirische Studien im Kontext manueller Arbeitsprozesse. Wiesbaden: Springer.

Sailer, M., Hense, J., Mandl, J., \& Klevers, M. (2014). Psychological perspectives on motivation through gamification. Interaction Design and Architecture Journal, 19, 28-37.

Sailer, M., Hense, J. U., Mayr, S. K., \& Mandl, H. (2017). How gamification motivates: An experimental study of the effects of specific game design elements on psychological need 
satisfaction. Computers in Human Behavior, 69(Supplement C), 371-380. https://doi.org/ 10.1016/j.chb.2016.12.033.

Sailer, M., \& Homner, L. (2020). The gamification of learning: A meta-analysis. Educational Psychology Review, 32(1), 77-112. https://doi.org/10.1007/s10648-019-09498-w.

Sailer, M., \& Sailer, M. (2020). Gamification of in-class activities in flipped classroom lectures. British Journal of Educational Technology. https://doi.org/10.1111/bjet.12948.

Sailer, M., Tolks, D., \& Mandl, H. (2019). Potenziale von Gamification: Empirische Befunde zum Einsatz in Schule und Unterricht. Computer + Unterricht, 115, 8-11.

Sanchez, D. R., Langer, M., \& Kaur, R. (2020). Gamification in the classroom: Examining the impact of gamified quizzes on student learning. Computers \& Education, 144(103666).

Schmidt-Kraepelin, M., Thiebes, A., Baumsteiger, S., \& Sunyaev, D. (2018). State of play: A citation network analysis of healthcare gamification studies. in research papers, 173 (S. 1-17). European Conference on Information Systems (ECIS2018), Portsmouth https://eci s2018.eu/published-ecis-2018-papers/. Zugegriffen: 16. Nov. 2020.

Singh, J. (2017/2019). Top gamification tools for moodle and other LMS (2019 Update). LMS Pulse. https://www.lmspulse.com/2017/top-6-plugins-for-gamification-in-moodle-gamifi cation/. Zugegriffen: 4. Nov. 2020.

Singhal, S., Hough, J., \& Cripps, D. (2019). Twelve tips for incorporating gamification into medical education. MedEdPublish, 8(3), 67.

Snow, R. E., \& Farr, M. J. (1987). Aptitude, learning, and instruction 3. cognitive and affective process analyses. Hillsdale: Erlbaum.

Snyder, K. D. (2003). Ropes, poles, and space: Active learning in business education. Active Learning in Higher Education, 4(2), (S. 159-167). https://doi.org/10.1177/146978740300 4002004.

Struyven, K., Dochy, F., Janssens, S., \& Gielen, S. (2006). On the dynamics of students' approaches to learning: The effects of the teaching/learning environment. Learning and instruction, 16(4), 279-294.

Tolks, D., Dadaczynski, K., \& Horstmann, D. (2018). Einführung in die Vergangenheit, Gegenwart und Zukunft von Serious Games (for Health). Prävention und Gesundheitsförderung, 13(4), 272-279. https://doi.org/10.1007/s11553-018-0667-9.

Tolks, D., \& Lampert, C. (2016). Abgrenzung von Serious Games zu anderen Lehr- und Lernkonzepten. In K. Dadaczynski, S. Schiemann, \& P. Paulus (Hrsg.), Gesundheit spielend fördern. Potenziale und Herausforderungen von digitalen Spieleanwendungen für die Gesundheitsförderung und Prävention (S. 191-217). Weinheim: Beltz Juventa.

Tolks, D., Lampert, C., Dadaczynski, K., Maslon, E., Paulus, P., \& Sailer, M. (2020). Spielerische Ansätze in Prävention und Gesundheitsförderung: Serious Games und Gamification. Bundesgesundheitsblatt - Gesundheitsforschung - Gesundheitsschutz, 63(6), 698-707. https://doi.org/10.1007/s00103-020-03156-1.

Tolks, D., Romeike, B., Ehlers, J. P., Kuhn, S., Kleinsorgen, C., Huber, J., Fischer, M. R., Bohne, C., \& Hege, I. (2020). The online Inverted Classroom Model (oICM). A blueprint to adapt the inverted classroom to an online learning setting in medical and health education. MedEdPublish, 9(1), 113.

Wang, H., \& Sun, C.-T. (2011). Game reward systems: Gaming experiences and social meanings. In Proceedings of the 2011 DiGRA International Conference: Think Design Play (S. 1-12). Utrecht School of the Arts. 
Werbach, K., \& Hunter, D. (2012). For the win: How game thinking can revolutionize your business. Philadelphia: Wharton Digital Press.

Xi, N., \& Hamari, J. (2019). Does gamification satisfy needs? A study on the relationship between gamification features and intrinsic need satisfaction. International Journal of Information Management, 46, 210-221. https://doi.org/10.1016/j.ijinfomgt.2018.12.002.

Zainuddin, Z. (2018). Students' learning performance and perceived motivation in gamified flipped-class instruction. Computers \& Education, 126, 75-88. https://doi.org/10.1016/j. compedu.2018.07.003.

Zainuddin, Z., Chu, S. K. W., Shujahat, M., \& Perera, C. J. (2020). The impact of gamification on learning and instruction: A systematic review of empirical evidence. Educational Research Review, 30(100326). https://doi.org/10.1016/j.edurev.2020.100326.

Zicherman, G., \& Cunningham, C. (2011). Gamification by design: Implementing game mechanics in web and mobile apps. Sebastopol: O’Reilly Media.

Open Access Dieses Kapitel wird unter der Creative Commons Namensnennung 4.0 International Lizenz (http://creativecommons.org/licenses/by/4.0/deed.de) veröffentlicht, welche die Nutzung, Vervielfältigung, Bearbeitung, Verbreitung und Wiedergabe in jeglichem Medium und Format erlaubt, sofern Sie den/die ursprünglichen Autor(en) und die Quelle ordnungsgemäß nennen, einen Link zur Creative Commons Lizenz beifügen und angeben, ob Änderungen vorgenommen wurden.

Die in diesem Kapitel enthaltenen Bilder und sonstiges Drittmaterial unterliegen ebenfalls der genannten Creative Commons Lizenz, sofern sich aus der Abbildungslegende nichts anderes ergibt. Sofern das betreffende Material nicht unter der genannten Creative Commons Lizenz steht und die betreffende Handlung nicht nach gesetzlichen Vorschriften erlaubt ist, ist für die oben aufgeführten Weiterverwendungen des Materials die Einwilligung des jeweiligen Rechteinhabers einzuholen.

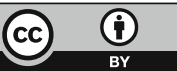

